\title{
Efeitos da poluição aérea e edáfica no sistema radicular de Tibouchina pulchra Cogn. (Melastomataceae) em área de Mata Atlântica: associações micorrízicas e morfologia ${ }^{1}$
}

\author{
SOLANGE C. MAZZONI-VIVEIROS ${ }^{2,3}$ e SANDRA F.B. TRUFEM ${ }^{2}$
}

(recebido: 13 de novembro de 2002; aceito: 19 de fevereiro de 2004)

\begin{abstract}
Effects of air and soil pollution on the root system of the Tibouchina pulchra Cogn. (Melastomataceae): arbuscular mycorrhizal associations and morphology) in Atlantic Forest area. The effects of atmospheric pollutants, originated from the industrial region of Cubatão, São Paulo State, were analysed on plants of Tibouchina pulchra from three areas with different types of pollutants. Root systems were used from passive and active monitoring specimens, and open-top-chambers (filtrated and no-filtrated air) specimens too. It was possible to evaluate the quantitative data from the root systems, the vesicular-arbuscular mycorrhizae (VAM) diversity, the mycorrhizal colonization percentage and the spores quantity in the rhizosphere soil. The results showed that in the most polluted area the specimens have: - increase in the mycorrhizal colonization percentages; - more VAM species diversity and quantity; - redution tendency in the root system development, especially in the wood roots length; - increase tendency in the fine roots quantity. These results demonstrate that the species studied is tolerant to the stress caused from the pollutants, with redution of the tolerance when the action of atmospheric pollutants takes place together the action of soil pollutants.
\end{abstract}

Key words - biomonitoring, mycorrhizal colonization, pollution, root system, VAM diversity

RESUMO - (Efeitos da poluição aérea e edáfica no sistema radicular de Tibouchina pulchra Cogn. (Melastomataceae): associações micorrízicas arbusculares e morfologia) em área de Mata Attântica. O efeito dos poluentes aéreos, oriundos do complexo industrial de Cubatão, Estado de São Paulo, foi analisado em plantas de Tibouchina pulchra de três áreas com diferentes níveis de poluição. Utilizaram-se sistemas radiculares de espécimes dos biomonitoramentos passivo e ativo, além daqueles das câmaras de topo aberto (ar filtrado e ar não filtrado). Dados quantitativos do sistema radicular, a diversidade de fungos micorrízicos arbusculares (FMAs), a porcentagem de colonização das raízes e a quantidade de esporos presentes no solo da rizosfera foram avaliados. Os resultados demonstraram haver nos indivíduos sujeitos aos maiores índices de poluição: - aumento da colonização micorrízica arbuscular; - maiores diversidade e abundância de espécies de FMAs; - tendência de redução no desenvolvimento do sistema radicular, principalmente em relação ao comprimento e às raízes lenhosas; - tendência de aumento na quantidade de raízes de pequeno calibre. Os resultados confirmaram a tolerância da espécie ao estresse causado pelos poluentes, demonstrando que essa tolerância pode ser reduzida quando a ação dos mesmos ocorre pelas vias aérea e edáfica.

Palavras-chave - biomonitoramento, colonização micorrízica, diversidade de FMAs, poluição, sistema radicular

\section{Introdução}

O município de Cubatão se encontra no litoral da cidade de São Paulo onde, em sua área e adjacências, se localizam indústrias petroquímicas, de fertilizantes, de cimento e outras igualmente poluidoras que se instalaram no local ao redor da década de 1950

1. Parte da Tese de doutorado de S.C. Mazzoni-Viveiros, vinculada ao Programa de Pós-graduação do Departamento de Botânica da Universidade de São Paulo.

2. Instituto de Botânica, Caixa Postal 4005, 01061-970 São Paulo, SP, Brasil.

3. Autor para correspondência: mazzoni-viveiros@uol.com.br aproveitando-se das facilidades do maior porto marítimo do país, o Porto de Santos, e dentro do lema "desenvolvimento a qualquer custo".

A localização desse pólo industrial é tal que os poluentes emanados pelas chaminés (material particulado, fluoretos, amônia, óxidos de nitrogênio, hidrocarbonetos, dióxido de enxofre, entre outros) são carreados pelos ventos que sopram em direção ao continente e acabam por encontrar a barreira física representada pela Serra do Mar. Essa barreira promove a condensação da água evaporada do mar que, por sua vez, se precipita na região como chuva ácida, ainda carregada de elementos particulados e outros que estavam em suspensão na atmosfera (Ab'Saber 1987).

A partir de 1984 passou-se a perceber visíveis danos à vegetação fanerogâmica, com morte de muitas árvores 
e conseqüentes deslizamentos de terra, acarretados pelo solo desnudo e as chuvas abundantes que ocorrem na região. A partir de 1988, finalmente, as autoridades constituídas passaram a voltar a atenção para a área, propondo a realização de estudos que visassem o entendimento dos fenômenos que atingiam a região, bem como a proposta de medidas para a recuperação da antiga exuberante vegetação de Mata Atlântica (SMA 1990)

Ressalta-se, no entanto, que a topografia da região permitiu que áreas contíguas sofressem diferentes níveis de impactos. Assim, o Vale do Rio Pilões tem sido relativamente protegido, em virtude da barreira física representada pela Serra do Mar e a direção predominante dos ventos ser contrária à sua área. A Reserva Biológica do Alto da Serra de Paranapiacaba recebe os ventos que varrem a área industrial e, em conseqüência, é atingida pelos agentes poluentes em suspensão. O Vale do Rio Moji é severamente poluído, em especial pelos materiais particulados e gases oriundos de indústrias diversas das proximidades (Klumpp et al. 2000a).

Dentre os vários estudos realizados, constatou-se que, dentre as plantas aparentemente tolerantes aos poluentes da região, encontravam-se espécies de Piperaceae, Urticaceae e Melastomataceae (Leitão Filho 1993). Dentre as espécies arbóreas a dominância relativa nas áreas poluídas parece ser das heliófilas, destacando-se as pertencentes às famílias Ericaceae, Moraceae e Melastomataceae, esta com maior valor de IVI (índice de valor de importância) e tendo como espécie dominante Tibouchina pulchra Cogn. (Pompéia 2000).

Com o aumento dos problemas ambientais, foram desenvolvidos métodos voltados para o seu monitoramento, utilizando-se, dentre outras possibilidades, plantas ou animais como bioindicadores (Ellenberg et al. 1991). Esses passam a apresentar reações específicas quando expostos a diferentes tipos de poluentes, fornecendo informações difíceis de serem obtidas e/ou quantificadas de outra forma (Flores 1987). Os biomonitoramentos, passivo e ativo, têm sido amplamente utilizados no Brasil nas últimas décadas para estudos diversos da qualidade do ar e efeito dos poluentes sobre as espécies vegetais (Flores 1987, Domingos et al. 1998, Klumpp et al. 2000a, b, MazzoniViveiros 2000). Estudos referentes às condições edáficas em áreas sujeitas à poluição atmosférica, especialmente na Serra do Mar em regiões próximas ao complexo industrial de Cubatão, têm sido também realizados (Mayer et al. 2000a, b).
Os fungos micorrízicos arbusculares (FMAs) são organismos simbiotróficos obrigatórios que se associam às raízes da maioria das plantas fanerogâmicas terrestres $(90 \%)$, sejam elas nativas ou de interesse agrícola. Nessa associação, o fungo penetra no córtex da raiz da planta, colonizando-o e aí formando estruturas densamente ramificadas, os arbúsculos. Externamente à raiz, o solo é colonizado pelas hifas, sendo explorado em maior volume e com maior eficiência do que as raízes o fazem. O micélio que explora o solo absorve água e sais minerais, particularmente $\mathrm{P}$, mas também $\mathrm{K}, \mathrm{S}, \mathrm{Ca}, \mathrm{Mg}$ dentre outros, e os transfere às plantas hospedeiras por intermédio dos arbúsculos. Em troca, a planta cede ao fungo compostos fotossintetizados, estabelecendo-se, assim, a relação mutualista (Read 1991, Morton 2001).

Estudos realizados em várias partes do mundo vêm demonstrando que os FMAs, além das relações nutricionais benéficas que conferem às plantas que os abrigam, de certa forma também participam conferindo-lhes algum tipo de proteção ou resistência a agentes causadores de estresses ambientais, como chuvas ácidas e metais pesados.

Os FMAs, por serem benéficos aos hospedeiros, exercem grande influência no restabelecimento da vegetação em áreas sob impacto ambiental, sendo estudados sob vários aspectos, como no caso de solos empobrecidos ou com déficit hídrico (Trufem 1990a, b, Trufem \& Viriato 1990), poluição atmosférica e/ou chuvas ácidas (Killham \& Firestone 1983, Trufem \& Malatinszky 1995), metais pesados (Gildon \& Tinker 1983a, b, Denny \& Ridge 1992), bem como deslizamentos (Day et al. 1987, Habte et al. 1988).

O presente trabalho tem como objetivo avaliar as associações micorrízicas arbusculares e a morfologia do sistema radicular em indivíduos de Tibouchina pulchra Cogn. (Melastomataceae) - oriundos de monitoramentos ativo e passivo - visando contribuir para o melhor entendimento dos efeitos dos poluentes atmosféricos vias direta (atmosfera) e indireta (solo) e oferecendo, assim, subsídios para programas de recuperação de áreas de Mata Atlântica sob o impacto de agentes semelhantes.

\section{Material e métodos}

Foram selecionados para o estudo exemplares de Tibouchina pulchra Cogn. (Melastomataceae), espécie nativa da Serra do Mar e conhecida pelo nome vulgar de "manacáda-serra". Essa espécie é encontrada tanto nas áreas mais protegidas da poluição como em áreas sob grande impacto, 
sendo considerada como tolerante e secundária (Leitão Filho 1993, Klumpp et al. 1994, Klockow et al. 1997, Klumpp et al. 2000a, b, Pompéia 2000).

As áreas selecionadas para o estudo, com diferentes níveis de poluição atmosférica, foram: - Vale do Rio Pilões ( $23^{\circ} 60^{\prime} \mathrm{S}$ e $46^{\circ} 36^{\prime} \mathrm{W}$ ), município de Cubatão, 40 a 150 metros de altitude, área a sudoeste do complexo industrial, recebendo pouca influência da poluição oriunda do mesmo; - Reserva Biológica do Alto da Serra de Paranapiacaba (23⒋ $\left.46^{\circ} 18^{\prime} \mathrm{W}\right)$, município de Santo André, 800 a 860 metros de altitude, localizada no alto da Serra em frente ao Vale do Rio Moji e, devido estar em local favorável à ação predominante dos ventos, é bastante atingida pelos poluentes secundários,

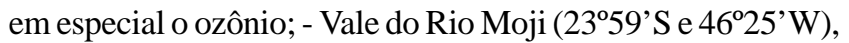
município de Cubatão, 20 a 250 metros de altitude, nas proximidades de indústrias de fertilizantes, cimento e siderúrgicas e, conseqüentemente, altamente poluído por particulados, fluoretos e compostos de enxofre e nitrogênio.

As condições ambientais e os dados do solo em cada uma das áreas selecionadas se encontram descritas nas tabelas 1 a 3 .

Para os biomonitoramentos foram avaliados indivíduos da espécie preexistentes nas áreas selecionadas (biomonitoramento passivo) e indivíduos jovens, envasados com solo de mata não sujeita à poluição atmosférica, introduzidos nas mesmas áreas ou submetidos à atmosfera de câmaras de topo aberto (biomonitoramento ativo) (Ellenberg et al. 1991, Klumpp et al. 2000a, b, Mazzoni-Viveiros 2000).

Para o biomonitoramento passivo foram utilizados os sistemas radiculares de indivíduos jovens de T. pulchra, com cerca de $30 \mathrm{~cm}$ de altura, coletados em fevereiro/1992 em cada uma das três áreas citadas, sendo 10 indivíduos para cada área.

No biomonitoramento ativo foram utilizados os sistemas radiculares de plantas jovens envasadas em cuja base foram acondicionadas caixas plásticas para atuarem como reservatório de água. Para cada área foram coletados dez sistemas radiculares de plantas mantidas em suporte de alumínio, cujas laterais foram utilizadas para colocação de anteparos contra o excesso de insolação, e expostas de fevereiro a maio/1993 às diferentes condições de poluição atmosférica de cada área em estudo. Nas câmaras de topo aberto, instaladas na Reserva de Paranapiacaba e cujas plantas permaneceram expostas durante o período de junho/1993 a janeiro/1994, foram coletados dez sistemas radiculares do lote mantido em câmara com sistema de filtragem e dez para aquela sem filtragem. As condições atmosféricas das câmaras estão descritas na tabela 4 , sendo que as mesmas apresentaram um acréscimo de $2,5^{\circ} \mathrm{C}$ na temperatura e $18 \%$ na umidade relativa, bem como uma redução de $25 \%$ a $30 \%$ de luminosidade, em relação ao ambiente externo.

Os sistemas radiculares das plantas do biomonitoramento passivo foram retirados do solo, com o auxílio de pá de jardineiro, até $20 \mathrm{~cm}$ de profundidade, acondicionando-os em sacos plásticos juntamente com o solo da rizosfera e, posteriormente, mantidos sob refrigeração (4 a $8^{\circ} \mathrm{C}$ ) até o momento do processamento. Na coleta de amostras do biomonitoramento ativo e das câmaras de topo aberto, as plantas foram desenvasadas, sendo que os sitemas radiculares e respectivos solos da rizosfera receberam o mesmo tratamento aplicado ao biomonitoramento passivo.

Para avaliação do porcentual de colonização por fungos micorrízicos arbusculares, alíquotas de raízes de cada uma das amostras (cerca de $0,5 \mathrm{~g}$ ) foram individualmente separadas do solo, lavadas em água corrente, fixadas em FAA $50 \% \mathrm{e}$, posteriormente, submetidas à coloração com fucsina ácida (Kormanik \& McGraw 1982), sendo a seguir avaliadas segundo a técnica da interseção de quadrantes (Giovannetti \& Mosse 1980). Esse material, quando pertinente, foi preparado entre lâmina e lamínula para observação ao microscópio fotônico.

Para o isolamento dos esporos de FMAs utilizou-se a técnica do peneiramento do solo $(100 \mathrm{~g})$ em via úmida

Tabela 1. Comparação dos valores médios de concentrações atmosféricas de $\mathrm{SO}_{2}, \mathrm{O}_{3}$ e $\mathrm{NO}_{2}$, temperatura, umidade relativa ${ }^{(1)} \mathrm{e}$ valores médios de precipitação ${ }^{(2)}$ nos diferentes sítios experimentais: Vale do Rio Pilões (Pilões), Reserva Biológica do Alto da Serra de Paranapiacaba (Reserva) e Vale do Rio Moji (Moji) (Klumpp et al. 2000b).

Table 1. Comparasion of means of $\mathrm{SO}_{2}, \mathrm{O}_{3}$ and $\mathrm{NO}_{2}$ atmospheric concentrations, temperature, relative humidity ${ }^{(1)}$ and means of precipitation ${ }^{(2)}$ in the experimental sites: "Vale do Rio Pilões" (Pilões), "Reserva Biológica do Alto da Serra de Paranapiacaba" (Reserva) and "Vale do Rio Moji" (Moji) (Klumpp et al. 2000b).

\begin{tabular}{lccccccc}
\hline Sítios & Período de medição & $\begin{array}{c}\mathrm{SO}_{2} \\
\left(\mu \mathrm{g} \cdot \mathrm{m}^{-3}\right)\end{array}$ & $\begin{array}{c}\mathrm{O}_{3} \\
\left(\mu \mathrm{g} \cdot \mathrm{m}^{-3}\right)\end{array}$ & $\begin{array}{c}\mathrm{NO}_{2} \\
\left(\mu \mathrm{g} \cdot \mathrm{m}^{-3}\right)\end{array}$ & $\begin{array}{c}\text { Temperatura } \\
\left({ }^{\circ} \mathrm{C}\right)\end{array}$ & $\begin{array}{c}\text { Umidade relativa } \\
(\%)\end{array}$ & $\begin{array}{c}\text { Precipitação } \\
(\mathrm{mm})\end{array}$ \\
\hline Pilões & $1991-1994$ & 6,7 & 29,8 & 7,5 & 21,2 & 86,8 & 3071 \\
Reserva & $1991-1995$ & 8,3 & 47,2 & 17,1 & 18,5 & 86,9 & 3368 \\
Moji & $1991-1995$ & 18,1 & 27,1 & 24,9 & 22,0 & 85,2 & 2432 \\
\hline
\end{tabular}

${ }^{(1)}$ Dados do Projeto Brasil/Alemanha (Klockow et al. 1997); data from Brazil/Germany Project (Klockow et al. 1997)

${ }^{(2)}$ Dados da Eletropaulo e DAEE; data from "Eletropaulo" and "DAEE" 
Tabela 2. Comparação dos valores médios de $\mathrm{pH}$ e de concentrações anuais de vários parâmetros químicos em solução de solo de amostras retiradas em diferentes profundidades (Prof.), entre 1991 e 1995, dos diferentes sítios experimentais: Vale do Rio Pilões (Pilões), Reserva Biológica do Alto da Serra de Paranapiacaba (Reserva) e Vale do Rio Moji (Moji) (Mayer et al. 2000b).

Table 2. Comparasion of means of $\mathrm{pH}$ values and several chemical parameters annual concentration in the soil solution from samples collected at varied depths (Prof.), between 1991 and 1995, of the experimental sites: "Vale do Rio Pilões" (Pilões), "Reserva Biológica do Alto da Serra de Paranapiacaba" (Reserva) and "Vale do Rio Moji" (Moji) (Mayer et al. 2000b).

\begin{tabular}{|c|c|c|c|c|c|c|c|c|c|c|c|c|c|c|c|}
\hline \multirow[b]{2}{*}{ Sítios } & \multirow[b]{2}{*}{$\begin{array}{l}\text { Prof. } \\
(\mathrm{cm})\end{array}$} & \multirow[b]{2}{*}{$\mathrm{pH}$} & \multicolumn{13}{|c|}{ Concentrações médias anuais $\left(\mu \mathrm{eq} . \mathrm{L}^{-1}\right)$} \\
\hline & & & $\begin{array}{l}\text { Ânions } \\
\text { totais }\end{array}$ & $\begin{array}{l}\text { Cátion } \\
\text { totais }\end{array}$ & $\mathrm{NO}_{3}-\mathrm{N}$ & $\mathrm{NH}_{4}-\mathrm{N}$ & $\mathrm{SO}_{4}-\mathrm{S}$ & $\mathrm{K}$ & $\mathrm{H}$ & $\mathrm{Ca}$ & $\mathrm{Mg}$ & $\mathrm{Al}$ & $\mathrm{Na}$ & $\mathrm{F}$ & $\mathrm{Cl}$ \\
\hline \multirow[t]{2}{*}{ Pilões } & 10 & 4.30 & 743 & 703 & 292 & 7 & 281 & 66 & 50 & 154 & 240 & 50 & 136 & 9 & 161 \\
\hline & 60 & 4.37 & 462 & 421 & 69 & 6 & 239 & 27 & 42 & 72 & 134 & 41 & 99 & 9 & 145 \\
\hline \multirow[t]{2}{*}{ Reserva } & a 10 & 3.50 & 532 & 505 & 101 & 6 & 349 & 4 & 316 & 43 & 20 & 53 & 63 & 11 & 71 \\
\hline & 60 & 3.59 & 487 & 400 & 63 & 6 & 295 & 2 & 256 & 19 & 6 & 82 & 28 & 11 & 118 \\
\hline \multirow[t]{2}{*}{ Moji } & 10 & 3.47 & 2528 & 2022 & 976 & 16 & 1170 & 50 & 339 & 606 & 382 & 378 & 260 & 60 & 322 \\
\hline & 60 & 3.57 & 2713 & 1552 & 940 & 7 & 1466 & 8 & 269 & 243 & 169 & 751 & 105 & 76 & 231 \\
\hline
\end{tabular}

(Gerdemann \& Nicolson 1963), seguindo-se centrifugação em sacarose $50 \%$ (v:v) para separação dos esporos (Daniels \& Skipper 1982). Os esporos de FMAs foram separados sob microscópio estereoscópico, quantificados e preparados entre lâmina e lamínula, com resina à base de álcool polivinílico e lactofenol (Trappe \& Schenck 1982). Tais lâminas foram observadas ao microscópio fotônico para fins de identificação (Schenck \& Perez 1988).

Para a avaliação morfométrica radicular, utilizou-se o material do biomonitoramento ativo e das câmaras de topo aberto, num total de 60 para o primeiro e 18 para o segundo. No ativo, as plantas foram expostas durante três meses às condições atmosféricas de cada área, em diferentes períodos, sendo dois lotes de março a setembro de 1992 e um de maio a agosto de 1993 (período seco), além de dois lotes de outubro a maio de 1993 (período chuvoso). Nas câmaras as plantas foram expostas de junho de 1993 a janeiro de 1994. Os sistemas foram, após lavagem em água corrente, fixados em FAA 70\% por 48 horas e mantidos, posteriormente, em álcool $70^{\circ} \mathrm{GL}$ ou submetidos à secagem para obtenção de peso seco. Foram determinados o comprimento e o diâmetro máximos, o número de raízes laterais lenhosas e a presença ou não da raiz principal facilmente identificada. Além disso, foi possível obter, em um dos lotes, o peso seco de cada um dos sistemas radiculares Os dados quantitativos foram analisados estatisticamente através do Programa SPSS-PC ${ }^{+}$, versão 4.0. Considerou-se, para tanto, a integração dos fatores área e período do ano, aplicando-se, de acordo com a necessidade, as provas de Duncan para a comparação múltipla entre as médias. As premissas da análise de variância foram verificadas através da prova de Shapiro-Wilks para a normalidade e pela prova de Lavene para a homogeneidade. Para os agentes ativos foram considerados duas estações (chuvosa e seca) e três diferentes graus, relacionados com os níveis de poluição das áreas em estudo (Pilões, Paranapiacaba e Moji). Para as câmaras de topo aberto, considerou-se apenas uma estação com dois diferentes níveis de qualidade do ar (ar filtrado e não filtrado).

Tabela 3. Comparação das concentrações médias anuais de elementos na serapilheira, entre 1991 e 1994, dos diferentes sítios experimentais: Vale do Rio Pilões (Pilões), Reserva Biológica do Alto da Serra de Paranapiacaba (Reserva) e Vale do Rio Moji (Moji), (Mayer \& Lopes 1997 apud Klockow et al. 1997).

Table 3. Comparasion of means of annual concentrations of litterfall elements, between 1991 and 1994, of experimental sites: "Vale do Rio Pilões" (Pilões), "Reserva Biológica do Alto da Serra de Paranapiacaba" (Reserva) and "Vale do Rio Moji" (Moji) (Mayer \& Lopes 1997 apud Klockow et al. 1997).

\begin{tabular}{|c|c|c|c|c|c|c|c|c|c|c|c|c|}
\hline \multirow[b]{2}{*}{ Sítios } & \multicolumn{12}{|c|}{ Concentrações médias anuais (mg.g ${ }^{-1}$ matéria seca) } \\
\hline & $\mathrm{N}$ & S & $\mathrm{P}$ & K & $\mathrm{Ca}$ & $\mathrm{Mg}$ & $\mathrm{Na}$ & $\mathrm{B}$ & $\mathrm{Cu}$ & $\mathrm{Fe}$ & $\mathrm{Mn}$ & $\mathrm{Zn}$ \\
\hline Pilões & 17,57 & 1,97 & 0,65 & 3,24 & 10,79 & 2,35 & 0,93 & 0,05 & 0,02 & 0,82 & 0,55 & 0,05 \\
\hline Reserva & 20,32 & 2,06 & 0,95 & 2,22 & 12,95 & 1,79 & 1,53 & 0,04 & 0,02 & 2,09 & 0,36 & 0,05 \\
\hline Moji & 18,91 & 2,19 & 1,03 & 1,59 & 10,89 & 1,23 & 1,60 & 0,04 & 0,01 & 2,71 & 0,15 & 0,04 \\
\hline
\end{tabular}


Tabela 4. Comparação entre os valores mínimos (min.), médios (méd.) e máximos (máx.) de $\mathrm{O}_{3}$ e $\mathrm{SO}_{2}$ atmosférico nas câmaras de topo aberto com os valores da atmosfera local e porcentagem de exclusão desses poluentes na câmara de ar filtrado $(\mathrm{AF}=$ ar filtrado; $\mathrm{ANF}=$ ar não filtrado $)$.

Table 4. Comparasion of minimum (min.), mean (méd.) and maximum (máx.) atmospheric $\mathrm{O}_{3}$ e $\mathrm{SO}_{2}$ values in the open top chambers, environment atmospheric values and pollutants exclusion percentage in the filtrated air open top chamber $(\mathrm{AF}=$ filtrated air; $\mathrm{ANF}=$ no filtrated air $)$.

\begin{tabular}{lrrrrrrrr}
\hline & \multicolumn{3}{c}{$\mathrm{O}_{3}(\mathrm{ppb})$} & & \multicolumn{3}{c}{$\mathrm{SO}_{2}(\mathrm{ppb})$} \\
\cline { 2 - 3 } \cline { 8 - 9 } & min. & méd. & máx. & & min. & méd. & máx. \\
\hline Câmara AF & 4,46 & 5,99 & 7,39 & & 0,35 & 1,27 & 2,80 \\
Câmara ANF & 17,31 & 19,20 & 21,95 & & 0,67 & 3,64 & 5,44 \\
Atmosfera local & 18,67 & 21,34 & 23,34 & & 3,29 & 4,34 & 4,47 \\
Exclusão na & 65,63 & 71,62 & 80,89 & & 67,98 & 80,81 & 89,36 \\
Câmara AF (\%) & 65 & & & & & \\
\hline
\end{tabular}

\section{Resultados e Discussão}

Os resultados da diversidade de FMAs e a porcentagem de colonização das raízes de T. pulchra das diferentes áreas e tratamentos encontram-se apresentadas na tabela 5 .

Verificou-se a ocorrência de 19 táxons de FMAs, sendo que dois deles possivelmente constituem espécies novas do gênero Acaulospora, que se fez representar com mais seis espécies. Seguem-se os gêneros Glomus Tul. \& Tul. e Scutellospora Walker \& Sanders com cinco espécies cada. O menos representado foi o gênero Gigaspora Gerd. \& Trappe emend. Walker \& Koske, com apenas uma espécie.

Os táxons verificados são de comum ocorrência em solos sob vegetação de Mata Atlântica, quer em áreas preservadas, como a Ilha do Cardoso (Trufem et al. 1994), quer em áreas sob impacto, como as regiões próximas a Cubatão (Trufem \& Malatinsky 1995, Trufem \& Viriato 1990). Como táxons menos freqüentes nesse tipo de ecossitema, pode-se apenas mencionar Glomus mosseae, que mais caracteristicamente ocorre em solos com $\mathrm{pH}$ variando do neutro ao levemente alcalino (Mosse et al. 1981), embora se registre a ocorrência da espécie em regiões sujeitas a chuvas ácidas e com metais pesados por Gildon \& Tinker (1983a, b). A espécie Scutellospora erythropa é mais característica de áreas arenosas, como dunas marítimas, e $S$. weresubiae tem sido menos freqüentemente isolada no país (Trufem 1990a).
Ainda em relação à diversidade verificada, Hayman \& Tavares (1985) e Wang et al. (1985) comentam que baixos valores de $\mathrm{pH}$ no solo favorecem a ocorrência de espécies dos gêneros Acaulospora, Gigaspora e Scutellospora, enquanto o gênero Glomus ocorre em valores de $\mathrm{pH}$ mais próximos da neutralidade. Os dados obtidos - ao confrontar as tabelas 2, 3 e 5 - reforçam tais afirmações para os gêneros Acaulospora e Scutellospora, enquanto Gigaspora teve sua ocorrência em solo menos ácido (Paranapiacaba). Quanto ao gênero Glomus, os resultados corroboram aqueles de Gildon \& Tinker (1983a, b), estando ausente no sítio com o valor mais elevado de pH (Pilões) e presente naqueles de valores menores (Pilões, Moji).

Quando se considera quantitativamente a ocorrência de esporos, Trufem et al. (1994) constataram, para plantas do litoral arenoso da Ilha do Cardoso (SP), correlação positiva com a temperatura, a precipitação e a insolação, ao mesmo tempo que a maior abundância de esporos ocorreu para os gêneros Acaulospora, Gigaspora e Scutellospora. A maior insolação e o aumento de temperatura do solo poderiam explicar o aumento no número de esporos e maior ocorrência do gênero Acaulospora nos sítios de Paranapiacaba e Moji, já que muitas clareiras existem nessas áreas; porém não explicam o maior número encontrado para a câmara de ar não filtrado, onde temperatura, precipitação e insolação foram iguais àqueles da câmara de ar filtrado.

O número de esporos obtidos, de maneira geral, foi baixo quando comparado com o valor médio de 50,04 esporos. $100 \mathrm{~g}^{-1}$ de solo da Mata Atlântica nativa da Ilha do Cardoso (Trufem 1990a), ou com os obtidos para mesma vegetação na Bahia, onde os autores verificaram variações entre 11 a 161 esporos. $100 \mathrm{~g}^{-1} \mathrm{de}$ solo (Santos \& Vinha 1982).

Considerando-se o número total de espécies encontrado em cada sítio experimental, os maiores valores foram obtidos para aqueles que apresentam níveis médio e alto de poluição, assim como para as rizosferas das plantas das câmaras de ar não filtrado. Ressalta-se, ainda, que além da diversidade maior ter ocorrido nos espécimes do biomonitoramento passivo dos sítios experimentais de Paranapacaba e Moji e nas câmaras de ar não filtrado, o número total de esporos em $100 \mathrm{~g}$ de solo foi crescente, acompanhando o aumento do nível de poluição atmosférica de Pilões a Moji.

A ocorrência de colonização heterogênea (hifas, arbúsculos, vesículas e/ou esporos) por FMAs em raízes da espécie foi crescente nos agentes passivos, 
Tabela 5. Diversidade de espécies de FMAs e porcentagem de colonização das raízes por FMAs em indivíduos de Tibouchina pulchra Cogn. oriundos do biomonitoramento passivo, em diferentes áreas da Serra do Mar, e das câmaras de topo aberto $(\mathrm{AF}=$ ar filtrado; $\mathrm{ANF}=$ ar não filtrado; Pilões = Vale do Rio Pilões; Reserva = Reserva Biológica do Alto da Serra de Paranapiacaba; Moji = Vale do Rio Moji $++=$ presença; - = ausência) .

Table 5. Species diversity of AMF and AMF root colonization percentage in Tibouchina pulchra Cogn. specimens natural of passive biomonitoring, from different Serra do Mar areas, and open top chambers (AF = filtrated air; ANF = no filtrated air; Pilões = "Vale do Rio Pilões"; Reserva = "Reserva Biológica do Alto da Serra de Paranapiacaba"; Moji = "Vale do Rio Moji"; + = presence; - = absence).

Táxon

\begin{tabular}{|c|c|c|}
\hline Biomonitoramento passivo & Câmaras & Ocorrência do \\
\hline Reserva & ANF & $(0 \%)$ \\
\hline
\end{tabular}

Acaulospora foveata Trappe \& Janos

A. longula Spain \& Schenck

A. mellea Spain \& Sckenck

A. scrobiculata Trappe

A. spinosa Walker \& Trappe

A. tuberculata Janos \& Trappe

Acaulospora sp. 1

Acaulospora sp. 2

Gigaspora gigantea Nicol. \& Gerd.

Glomus etunicatum Becker \& Gerd.

G. invermayum Hall

G. macrocarpumTul. \& Tul.

G. microcarpumTul. \& Tul.

G. mosseae (Nicol. \& Gerd.) Gerd. \& Trappe

Scutellospora erythropa (Koske \& Walker) Walker

S. heterogama (Nicol. \& Gerd.) Walker \& Sanders

S. persica (Koske \& Walker) Walker \& Sanders

S. verrucosa (Koske \& Walker) Walker \& Sanders

S. weresubuiae Koske \& Walker

Total de táxons

№ total de esporos em $100 \mathrm{~g}$ solo

$\begin{array}{rrrrrr}+ & + & + & + & + & 100 \\ - & + & + & - & + & 60 \\ - & + & + & - & - & 40 \\ - & - & - & + & + & 40 \\ - & - & - & - & + & 20 \\ + & + & + & - & - & 60 \\ + & + & + & - & - & 60 \\ - & + & - & - & - & 20 \\ + & - & - & - & - & 20 \\ - & + & - & - & + & 40 \\ - & - & - & + & + & 40 \\ - & + & - & + & + & 60 \\ - & - & + & - & + & 40 \\ - & - & - & + & + & 40 \\ - & - & - & - & + & 20 \\ - & - & - & + & + & 40 \\ - & - & + & - & - & 20 \\ - & - & - & + & - & 20 \\ - & - & - & + & - & 20 \\ 4 & 8 & 7 & 8 & 11 & \\ 7 & 32 & 40 & & & \end{array}$

Colonização (\%)

Biomonitoramento passivo

$37 \quad 44$

Biomonitoramento ativo

$25 \quad 55$

50

$25 \quad 23 \quad 30$

acompanhando o aumento do nível de poluição, da mesma forma que nas câmaras de topo aberto, onde a maior colonização foi obtida naquela de ar não filtrado. Nos agentes ativos, no entanto, o maior grau foi observado no sítio medianamente poluído de Paranapiacaba.

Levando-se em consideração os elementos químicos dos solos de cada sítio experimental (tabelas 2, 3), os menores valores de $\mathrm{pH}$, bem como altas concentrações de $\mathrm{Al}, \mathrm{S}, \mathrm{Fe}, \mathrm{Ca}, \mathrm{Mg}, \mathrm{C}, \mathrm{N}$ e ânions e cátions totais, ocorrem nos sítios experimentais com os maiores índices de poluição atmosférica (Reserva e
Moji), principalmente quando se analisa a dinâmica de tais elementos na solução de solo, levando-se em conta sua importação e exportação (Mayer et al. 2000a, b).

As micorrizas conferem à planta hospedeira vantagens, como aumento da capacidade de absorção de água e íons e transporte de micro e macronutrientes via hifas que interligam as plantas infectadas (Duin et al. 1991). A micorrização é inibida, geralmente, em condições de elevada fertilidade, sendo que dentre os macronutrientes são o nitrogênio $(\mathrm{N})$ e o fósforo $(\mathrm{P})$ que exercem maior influência; tais elementos agem indiretamente via metabolismo da planta hospedeira, 
estabelecendo mecanismo de auto-regulação. Devido ao fato das raízes, na mata, se desenvolverem de modo muito emaranhado, sugeriu-se que a colonização de raízes por hifas seria, nesse caso, mais eficiente para a garantia do fluxo de nutrientes (Francis et al. 1986).

A associação micorrízica atinge seu máximo de eficiência quando a concentração de fósforo $(\mathrm{P})$ no solo aumenta e a troca de nutrientes entre o fungo e a planta hospedeira atinge um nível ótimo (Schubert \& Hayman 1986). Em solos ricos, as raízes podem suprir a planta com fósforo sem a necessidade desse tipo de associação, sendo que em solos com níveis muito altos de fósforo tal associação pode ser baixa (Duin et al. 1991).

Leitão Filho (1993) afirma que, apesar do solo do sítio experimental do Vale do Rio Moji apresentar maior quantidade de fósforo total, a quantidade disponível de tal elemento é inferior àquela do Vale do Rio Pilões. Domingos et al. (2000) e Mayer et al. (2000a, b) apresentam resultados para os mesmos sítios experimentais do presente trabalho, onde ressaltam a alta acidez da solução do solo do Vale do Rio Moji, concentrações elevadas de alumínio, sulfato, nitrato, fluoreto e cálcio, além do fato de haver intenso processo de intemperização do solo, pela ação de ácidos e substâncias acidificantes, como $\mathrm{SO}_{4}$ e F. Tal processo causa liberação do $\mathrm{Al}$ dos minerais do solo, promovendo a dissolução do $\mathrm{Ca}$ e $\mathrm{Mg}$, depositados como particulados, e a liberação de $\mathrm{NO}_{3}$, resultando em níveis críticos de elementos nutricionais na zona radicular. Tais aspectos, como baixa disponibilidade de fósforo e de nutrientes, explicariam a maior porcentagem de associação na área mais poluída do Vale do Rio Moji.

Os dados, de maneira geral, sugerem aumento no número de esporos e na diversidade de espécies no solo em áreas sob o impacto da poluição atmosférica para o ecossistema em questão, confirmando os dados obtidos por Vilariño et al. (1992), que ressaltam a ocorrência do aumento de esporulação nos casos em que a parte aérea da planta hospedeira se encontra estressada. Confirmam, também, os dados obtidos por Trufem \& Malatinsky (1995) que afirmam que as espécies de Melastomataceae apresentam fatores que predispõem os FMAs de suas rizosferas a apresentarem esporulação mais abundante em área submetida à poluição atmosférica, do que as verificadas em plantas de outras famílias botânicas da mesma área.

Alguns estudos apontam para o fato que os FMAs podem causar aumento no teor de metais pesados nas plantas hospedeiras (Killham \& Firestone 1983), enquanto outros demonstram que tais fungos podem conferir maior grau de tolerância da planta aos metais
(Koslowsky \& Boerner 1989). O aumento de colonização na rizosfera de Tibouchina pulchra, em áreas mais afetadas pela ação dos poluentes atmosféricos, confirma as suspeitas de que a associação micorrízica pode atuar no decréscimo dos efeitos fitotóxicos dos metais no interior da planta (Schüepp et al. 1987), já que, segundo Leitão Filho (1993), a espécie é a que apresenta os maiores valores para os parâmetros fitossociológicos da área, como IVI (índice do valor de importância) e IVC (índice do valor de cobertura). Tal associação parece ser, portanto, muito importante para a revegetação de áreas degradadas.

Segundo Koske \& Halvorson (1981) e Koske (1987), os FMAs desempenham importante papel no estabelecimento e no processo de sucessão vegetal em dunas, já que aumentam a capacidade de absorção de nutrientes e de água pelas raízes, além de contribuírem para melhor estabilização do substrato. Considerando o exposto por Leitão Filho (1993), segundo o qual o Vale do Rio Moji apresenta evidente desequilíbrio ecológico, fazendo com que o processo de sucessão não se complete e volte ciclicamente para os seus estádios iniciais, e o fato de nesse sítio experimental ter ocorrido a maior quantidade de esporos (tabela 5), sugere-se que também na mata tais fungos estejam procurando desempenhar seu papel no processo sucessional.

Comparando-se os resultados obtidos no presente trabalho com aqueles obtidos por Trufem (1990a) para as rizosferas do gênero Tibouchina na vegetação da Ilha do Cardoso, pode-se afirmar que a diversidade foi maior em dois (Pilões e Moji) dos três sítios experimentais do presente estudo, já que naquela região somente cinco espécies pertencentes a três gêneros foram encontradas (Acaulospora, Sclerocystis e Glomus). Considerando-se o exposto por Giovannetti \& Gianinazzi-Pearson (1994), que afirmam que a conservação e utilização eficiente da biodiversidade do grupo de fungos responsáveis pela formação de micorrizas arbusculares é fator indispensável para a sustentabilidade da estrutura da comunidade vegetal, acredita-se que tais sítios ainda se encontram com nível adequado de biodiversidade dos FMAs e, portanto, sob esse ponto de vista, com capacidade de participarem no processo de revegetação.

O comprimento máximo apresentado pelo sistema radicular (tabela 6) evidenciou valores médios maiores na estação seca para todos os sítios experimentais, enquanto na chuvosa o menor valor foi para o sítio com maior nível de poluição, o Vale do Rio Moji, com diferenças significativas. Para as plantas das câmaras de topo aberto, no entanto, a maior média ocorreu para 
Tabela 6. Valores mínimos (min.), médios (méd.) e máximos (máx.0) para os dados referentes ao sistema radicular dos espécimes do biomonitoramento ativo e das câmaras de topo aberto. Resultados da análise de variância pela prova de Duncan na comparação entre os diferentes ambientes, os diferentes graus de poluição nas épocas chuvosa e seca e os diferentes graus de poluição nas câmaras. $(\mathrm{RP}=$ Vale do Rio Pilões; $\mathrm{PP}=$ Reserva de Paranapiacaba; $\mathrm{VM}=$ Vale do Rio Moji; $\mathrm{AF}=$ ar filtrado; $\mathrm{ANF}=$ ar não filtrado; $\mathrm{EC}$ = época chuvosa; ES = época seca; (1) = nível baixo de poluição; (2) = nível médio de poluição; (3) = nível alto poluição; $\mathrm{p}=$ probabilidade de erro; $\mathrm{s}=$ significativo; $\mathrm{ns}$ = não significativo; $\#$ = difere de forma significativa; $+=$ = resultado obtido).

Table 6. Minimum (min.), mean (méd.) and maximum (máx.) values for root system references data from active biomonitoring and open top chambers specimens. Duncan test results comparing the different environments, the varied loads of air pollution at rainy and dry periods and the varied loads of air pollution in th open top chambers. (RP = "Vale do Rio Pilões"; PP = "Reserva de Paranapiacaba"; $\mathrm{VM}=$ "Vale do Rio Moji"; $\mathrm{AF}$ = filtrated air; $\mathrm{ANF}=$ no filtrated air; $\mathrm{EC}$ = rainy period; $\mathrm{ES}$ = dry period; $(1)=$ pollution low load; $(2)=$ pollution mean load; $(3)=$ pollution high load; $p=$ error probability; $s=$ significative; ns = no significative; $\#=$ significative difference + + = result obtained $)$.

\begin{tabular}{|c|c|c|c|c|c|c|c|c|c|c|c|c|}
\hline & \multicolumn{3}{|c|}{ Comprimento máximo (cm) } & \multicolumn{3}{|c|}{ Número de raízes } & \multicolumn{3}{|c|}{ Matéria seca $(\mathrm{g})$} & \multicolumn{3}{|c|}{ Diâmetro máximo } \\
\hline & $\min$. & méd. & máx. & min. & méd. & máx. & $\min$. & méd. & máx. & $\min$. & méd. & máx. \\
\hline \multicolumn{13}{|c|}{ Biomonitoramento Ativo } \\
\hline $\mathrm{RP}(1) \mathrm{EC}$ & 17,00 & 31,60 & 47,00 & 06 & 11 & 15 & 2,17 & 3,90 & 6,26 & 4,50 & 7,15 & 9,00 \\
\hline $\mathrm{RP}(1) \mathrm{ES}$ & 31,00 & 46,30 & 60,00 & 04 & 10 & 17 & 2,12 & 3,81 & 5,15 & 3,50 & 6,85 & 14,00 \\
\hline $\mathrm{PP}(2) \mathrm{EC}$ & 18,00 & 34,20 & 45,00 & 05 & 09 & 13 & 1,74 & 3,37 & 5,23 & 6,00 & 8,05 & 11,50 \\
\hline $\mathrm{PP}(2) \mathrm{ES}$ & 32,00 & 38,80 & 48,00 & 05 & 07 & 10 & 1,34 & 3,76 & 5,88 & 3,50 & 6,35 & 10,00 \\
\hline $\mathrm{VM}(3) \mathrm{EC}$ & 22,00 & 30,70 & 42,00 & 07 & 10 & 14 & 2,14 & 3,34 & 5,68 & 5,50 & 8,85 & 13,00 \\
\hline $\mathrm{VM}(3) \mathrm{ES}$ & 29,00 & 40,00 & 50,00 & 05 & 07 & 10 & 1,07 & 3,74 & 12,03 & 5,00 & 7,05 & 11,00 \\
\hline \multicolumn{13}{|l|}{ Câmaras (C) } \\
\hline $\mathrm{AF}$ & 19,50 & 36,06 & 47,00 & 06 & 11 & 15 & 4,64 & 14,26 & 23,03 & 12,00 & 14,05 & 16,50 \\
\hline ANF & 20,00 & 42,50 & 70,00 & 08 & 14 & 21 & 6,27 & 10,68 & 13,91 & 11,00 & 12,38 & 15,00 \\
\hline \multicolumn{13}{|c|}{ Comparação entre os ambientes } \\
\hline $\mathrm{S}$ & \multicolumn{3}{|c|}{$\mathrm{EC} \# \mathrm{C} / \mathrm{EC} \# \mathrm{ES}$} & \multicolumn{3}{|c|}{ EC \# ES \#C } & \multicolumn{3}{|c|}{$\mathrm{C} \# \mathrm{EC} / \mathrm{C} \# \mathrm{ES}$} & \multicolumn{3}{|c|}{$\mathrm{EC} \# \mathrm{C} / \mathrm{ES} \# \mathrm{C}$} \\
\hline $\mathrm{ns}$ & \multirow{2}{*}{\multicolumn{3}{|c|}{0,0004}} & \multirow{2}{*}{\multicolumn{3}{|c|}{0,0000}} & & & & \multirow{2}{*}{\multicolumn{3}{|c|}{0,0000}} \\
\hline $\mathrm{P}$ & & & & & & & \multicolumn{3}{|c|}{0,0000} & & & \\
\hline \multicolumn{13}{|c|}{$\begin{array}{l}\text { Comparação entre diferentes graus de poluição - época chuvosa } \\
\text { S }\end{array}$} \\
\hline $\mathrm{ns}$ & \multicolumn{3}{|c|}{+} & \multicolumn{3}{|c|}{+} & \multicolumn{3}{|c|}{+} & \multicolumn{3}{|c|}{+} \\
\hline $\mathrm{P}$ & \multicolumn{3}{|c|}{0,66} & \multicolumn{3}{|c|}{0,16} & \multicolumn{3}{|c|}{0,41} & \multicolumn{3}{|c|}{0,90} \\
\hline \multicolumn{13}{|c|}{ Comparação entre diferentes graus de poluição - época seca } \\
\hline $\mathrm{S}$ & \multicolumn{6}{|c|}{ RP \# PP / RP \# VM } & & \\
\hline $\mathrm{ns}$ & \multirow{2}{*}{\multicolumn{3}{|c|}{0,04}} & & + & & & + & & & + & \\
\hline $\mathrm{p}$ & & & & & 0,79 & & & 0,06 & & & 1,00 & \\
\hline Comparação entre & entes grau & aus de po & luição - cât & aras & & & & & & & & \\
\hline $\mathrm{s}$ & & & & & \# ANF & & & & & & & \\
\hline $\mathrm{ns}$ & & + & & & & & & + & & & + & \\
\hline $\mathrm{p}$ & & 0,29 & & & 0,00 & & & 0,11 & & & 0,10 & \\
\hline
\end{tabular}

as da câmara de ar não filtrado. Ressalta-se, ainda, que a maior diferença entre o valor mínimo e o máximo ocorreu nas plantas da câmara de ar não filtrado. Pela análise realizada observa-se que tal fator apresentou variação significativa nos agentes ativos, quando os diferentes graus de poluição foram considerados na estação seca, ou seja, o comprimento da raiz na área menos poluída diferiu dos outros dois sítios mais poluídos, apresentando valores maiores naquele menos poluído do Vale do Rio Pilões. Outra variação significativa ocorreu quando foi efetuada a comparação entre período chuvoso $\times$ período seco $\times$ câmaras, sendo que neste caso os dados obtidos para a estação chuvosa diferem significativamente daqueles da estação seca e das 
câmaras, com valores sempre superiores nas plantas das câmaras.

O número de raízes laterais lenhosas do sistema radicular atingiu médias mais elevadas no sítio de Pilões e na câmara de ar não filtrado, sendo que os menores valores foram obtidos no sítio com o nível médio de poluição, o da Reserva do Alto da Serra de Paranapiacaba, e na câmara de ar filtrado. A maior diferença entre o valor mínimo e máximo encontrado para tal fator ocorreu na câmara de ar não filtrado e na estação seca no sítio de Pilões. A análise apresentou variação significativa entre a câmara de ar filtrado e não filtrado, com valores maiores nessa última. Quando somente os ambientes foram considerados houve variação significativa entre os dados nas estações chuvosa e seca e, também, nas câmaras. Não houve nenhuma variação significativa, porém, quando os diferentes graus de poluição foram comparados.

$\mathrm{O}$ peso da matéria seca (PS) do sistema radicular apresentou valores médios menores, tanto na estação chuvosa como na seca, para o sítio de Moji. Nas câmaras de topo aberto a menor média ocorreu na de ar não filtrado. As maiores diferenças entre os valores mínimos e máximos ocorreram na estação seca no sítio de Moji e na câmara de ar filtrado. A análise multivariada/ estatística apontou variação significativa apenas quando os diferentes ambientes foram considerados, ou seja, os valores das câmaras diferem significativamente daqueles das estações chuvosa e seca dos agentes ativos.

Em relação ao diâmetro máximo do sistema radicular, as maiores médias ocorreram na estação chuvosa nos sítios de Pilões e Moji e na câmara de ar filtrado, enquanto na estação seca os menores valores ocorreram no sítio de Paranapiacaba. A diferença maior apresentada entre os valores mínimos e máximos ocorreu na estação seca do sítio de Pilões e a menor nas câmaras de topo aberto. A análise indicou variação significativa somente entre os ambientes, ou seja, os valores das câmaras diferem significativamente daqueles da estação chuvosa e da seca dos agentes ativos, sendo que os diferentes graus de poluição dos tratamentos não diferem entre si.

Quanto ao aspecto morfológico encontrado nos sistemas radiculares dos agentes ativos e das plantas das câmaras de topo aberto, observou-se variação nas formas apresentadas dentro do mesmo sítio experimental e notou-se que nos diferentes lotes, tanto no período chuvoso como no período seco, a presença de raízes finas é maior nos agentes ativos dos sítios de Pilões e Moji. O mesmo ocorreu para os sistemas radiculares das plantas da câmara de ar não filtrado, dificultando, inclusive, a visualização das raízes mais internas. Essa variação de formas apresentada nos sistemas radicular em cada caso pode estar relacionada à grande produção de raízes finas, uma vez que as mesmas têm sido consideradas as responsáveis por crescimentos desordenados do sistema radicular (VavoulidouTheodorou \& Babel 1986).

Correlacionando tais dados com aqueles já descritos para os FMAs, sabe-se que a morfologia da raiz afeta tal associação, bem como a taxa de mortalidade da raiz influencia a heterogeneidade na formação da micorriza, sendo que a morte gradual da raiz estimula a esporulação, enquanto a morte repentina estimula a formação de hifas (Abbott et al. 1992, Janos 1992). Para que a associação se estabeleça, inclusive, é importante que as raízes finas apresentem o meristema apical íntegro, sendo que uma vez estabelecida a associação esta pode, também, estimular a formação de raízes curtas (Blaschke 1986). Blasius et al. (1985) chegam a propor que se procure relacionar a densidade de ramificação com a frequiência da associação micorrízica.

Considerando a maior ocorrência de colonização por hifas e o maior número de esporos no solo do sítio de Moji, parece haver perfeita integração de tais resultados com o aspecto apresentado pelo sistema radicular, com maior número de raízes finas. A grande quantidade de esporulação pode, inclusive, estar relacionada à morte gradual das raízes, que pode ser esperada pela ação do solo de área extremamente afetada pela poluição. O fato, ainda, de existir heterogeneidade na forma de colonização (hifas, vesículas, arbúsculos e esporos) pode refletir na taxa de mortalidade da raiz, como exposto por Janos (1992), podendo, inclusive, ser o fator causador do aumento de diâmetro do sistema radicular, tendência observada nas áreas mais poluídas (tabela 6).

A maior quantidade de raízes finas pode estar relacionada com as necessidades nutricionais da planta, já que tais raízes são as maiores responsáveis pelo suprimento de nitrogênio em angiospermas arbóreas (Mclaugherty et al. 1982). Pode, ainda, estar relacionado com a acidificação do solo, já que em solos fortemente ácidos há aumento da taxa de mortalidade nas raízes mais grossas, sendo que a proporção de raízes vivas e mortas está relacionada à relação $\mathrm{Ca} / \mathrm{Al}$ no interior da raiz, esta bem menor no solo e na água de solo nas áreas poluídas consideradas, conforme tabela 2 (Puhe et al. 1986).

Os dados demonstram que há aumento da colonização micorrízica arbuscular e maiores 
diversidade e abundância de espécies de FMAs sob solos ácidos em indivíduos da espécie T. pulchra sujeitos à poluição atmosférica. Evidenciou-se, também, tendência de redução do desenvolvimento de raízes lenhosas e aumento da quantidade de raízes finas (não lenhosas). Tais resultados reafirmam ser a espécie tolerante aos poluentes, com tolerância reduzida quando a ação de tais poluentes ocorre pelas vias aérea e edáfica. O uso, portanto, da espécie em reflorestamento de áreas de mata atlântica degradadas deve incluir medidas de recuperação do solo para que sejam bem sucedidas.

\section{Referências bibliográficas}

ABBOTT, L.K., ROBSON, A.D., JASPER, D.A. \& GAZEY, C. 1992. What is the role of VA mycorrhizal hyphae in soil? In Mycorrhizas in ecosystems (D.J. Read, D.H. Lewis, A.H. Fitter \& I.J. Alexander, eds.). C.B.A. International, Cambridge, p.37-41.

AB'SABER, A. 1987. A Serra do Mar na região de Cubatão: avalanches de janeiro de 1985. A ruptura do equilíbrio ecológico na Serra de Paranapiacaba e a poluição industrial. Aciesp 54:74-116.

BLASCHKE, H. 1986. Studies on the developemnt mycorrhizal fine roots of Norway spruce in declining forests. Forstwissenschaftliches Centralblatt 105:477-487

BLASIUS, D., KOTTKE, I. \& OBERWINKLER, F. 1985. Quality assessment of spruce roots from damage stands. Forstwissenschaftliches Centralblatt 104:318-325.

DANIELS, B.A. \& SKIPPER, H.D. 1982. Methods for the recovery and quantitative estimation of propagules from soil. In Methods and principles of mycorrhizal research (N.C. Schenck, ed.). The American Phytopathological Society, St. Paul, p.29-35.

DAY,L.D., SYLVIA, D.M. \& COLLINS, M.E. 1987. Interactions among vesicular arbuscular mycorrhizas, soil and landscape position. Soil Science Society American Journal 51:635-639.

DENNY, H. \& RIDGE, I. 1992. Mycorrhizal amelioration of metal toxicity to plants. In Mycorrhizas in ecosystems (D.J. Read, D.H. Lewis, A.H. Fitter \& I.J. Alexander, eds.). C.B.A. International, Cambridge, p.376.

DOMINGOS, M., KLUMPP, A. \& KLUMPP, G. 1998. Air pollutants impact on the Atlantic forest in the Cubatão region, São Paulo, Brazil. Ciência e Cultura 50:230-236.

DOMINGOS, M., LOPES, M.I.M.S. \& STRUFFALDI-DEVUONO, Y. 2000. Nutrient cycling disturbance in Atlantic Forest sites affected by air pollution coming from the industrial complex of Cubatão, Southeast Brazil. Revista Brasileira de Botânica 23:77-85.
DUIN, W.E. VAN, GRIFFIOEN, W.A.J. \& IETSWAART, J.H. 1991. Ocurrence and function of micorrhiza in environmentally stressed soils. In Ecological responses to environmental stresses (J. Rozema \& J.A.C. Verkleij, eds.). Kluwer Academic Publishers, Dordrecht, p.114-123.

ELLENBERG,H., ARNDT,U.,BRETTHAUER, R., RUTHSATZ, B. \& STEUBING, L. 1991. Biological monitoring: signals from the environment. Deutsches Zentrum für Entwicklungtechnologien - Gate, Eschborn.

FLORES, F.E.V. 1987. O uso de plantas como bioindicadores de poluição no ambiente urbano-industrial: experiências em Porto Alegre, RS, Brasil. Tübinger Geographische Studien 96:79-86

FRANCIS, R., FINLAY, R.D. \& READ, D.J. 1986. Vesicular arbuscular mycorrhizas in natural vegetation systems. IV. Transfer of nutrients in inter and intra-specific combinations of host plants. New Phytologist 102:103-111.

GERDEMANN, J.W. \& NICOLSON, T.H. 1963. Spores of mycorrhizal Endogone species extracted from soil by wet sieving and decanting. Transactions of the British Mycological Society 46:235-244.

GILDON, A. \& TINKER, P.B. 1983a. Interactions of VAM infection and heavy metal in plants. I. Effects of heavy metals on development of VAM. New Phytologist 93:241-261.

GILDON, A. \& TINKER, P.B. 1983b. Interactions of VAM infection and heavy metal in plants. II. The effects of infection on uptake of copper. New Phytologist 93:263-268.

GIOVANNETTI, M. \& GIANINAZZI-PEARSON, V. 1994. Biodiversity in arbuscular mycorrhizal fungi. Mycology Research 98:705-715.

GIOVANNETTI, M. \& MOSSE, B. 1980. An evaluation of techniques for measuring vesicular-arbuscular infection in roots. New Phytologist 84:489-500.

HABTE, M., FOX, R.L., AZIZ, T. \& EL-SWAIFY, A. 1988. Interaction of vesicular arbuscular mycorrhizal fungi with erosion in an oxisol. Applied and Environmental Microbiology 54:945-950.

HAYMAN, D.S. \& TAVARES, M. 1985. Plant growtth responses to vesicular arbuscular mycorrhiza: XV. Influence of soil $\mathrm{pH}$ on the symbiotic efficiency of different endophytes. New Phytologist 100:367-377.

JANOS, D.P. 1992. Heterogeneity and scale in tropical vesicular-arbuscular mycorrhiza formation. In Mycorrhizas in ecosystems (D.J. Read, D.H. Lewis, A.H. Fitter \& I.J. Alexander, eds.). C.B.A. International, Cambridge, p.276-282.

KILLHAM, K. \& FIRESTONE, M.K. 1983. Vesicular arbuscular mycorrhizal mediation of grass response to acidic and heavy metals depositions. Plant Soil 72:39-48. 
KLOCKOW, D., TARGA, H. \& VAUTZ, W. (eds.). 1997. Air pollution and vegetation damage in the tropics - the Serra do Mar as an example, Final Report, Secretaria do Meio Ambiente/Geesthacht GKSS, São Paulo/Dortmund.

KLUMPP, A., KLUMPP, G. \& DOMINGOS, M. 1994. Plants as bioindicators of air pollution at the Serra do Mar near the industrial complex of Cubatão, Brazil. Environmental Pollution 85:109-116.

KLUMPP, A., DOMINGOS, M. \& PIGNATA, M.L. 2000a. Air pollution and vegetation damage in South America state of knowledge and perspectives. In Environmental Pollution and plant responses (S.B. Agrawal \& M. Agrawal, eds.). Lewis Publishers, Boca Raton, p.111-136.

KLUMPP, G., FURLAN, C.M., DOMINGOS, M. \& KLUMPP, A. 2000b. Response of stress indicators and growth parameters of Tibouchina pulchra Cogn. exposed to air and soil pollution near the industrial complex of Cubatão, Brazil. The Science of the Total Environment 246:79-91.

KORMANIK, P.P. \& MCGRAW, A.C. 1982. Quantification of vesicular-arbuscular mycorrhizae in plant roots. In Methods and principles of mycorrhizal research. (N.C. Schenck, ed.).The American Phytopathological Society, St. Paul, p.37-45.

KOSKE, R.E. 1987. Distribution of vesicular-arbuscular mycorrhiza, fungi along a latitudinal temperature gradient. Mycologia 79:55-68.

KOSKE, R.E. \& HALVORSON, W.L. 1981. Ecological studies of vesicular arbuscular mycorrhiz in a barrier sand dune. Canadian Journal of Botany 59:1413-1422.

KOSLOWSKY, D.D. \& BOERNER, R.E.J. 1989. Interactive effects of aluminum, phosphorus, and mycorrhizae on growth and nutrient uptake of Panicum virgatum L. (Poaceae). Environmental Pollution 61:105-125.

LEITÃO FILHO, H.F. (ed.). 1993. Ecologia da Mata Atlântica em Cubatão. Ed. Unesp/Ed. Unicamp, São Paulo, Campinas.

MAYER, R., LIESS, S., LOPES, M.I.M.S. \& KREUTZER, K. 2000a. Atmospheric pollution in a tropical rain forest: effects of deposition upon biosphere and hydrosphere. I. Concentrations of chemicals. Water, Air, and Soil Pollution 121:59-78.

MAYER, R., LIESS, S., LOPES, M.I.M.S. \& KREUTZER, K. 2000b. Atmospheric pollution in a tropical rain forest: effects of deposition upon biosphere and hydrosphere. II. Fluxes of chemicals and element budgets. Water, Air, and Soil Pollution 121:79-92.

MAZZONI-VIVEIROS, S.C. 2000. Diversificação do uso da anatomia: poluição atmosférica na Mata Atlântica. In Tópicos atuais em Botânica (T.B. Cavalcanti \& B.M.T. Walter, eds.). Embrapa/Sociedade Botânica do Brasil, Brasília, p.101-106.

MCLAUGHERTY, C.A., ABER, J.D. \& MELILLO, J.M. 1982. The role of fine roots in th organic matter and nitrogen budgets of two forested ecosystems. Ecology 63:1481-1490.

MORTON, J. 2001. http://www.invam.caf.wvu.edu. (acesso em 02/2001).
MOSSE, B., STRIBLEY, D.P. \& LE TACON, F. 1981. Ecology of mycorrhizae and mycorrhizal fungi. Advances in Microbiology Ecology 5:137-210.

POMPÉIA, S.L. 2000. Resposta das plantas nativas brasileiras à poluição. In Ecofisiologia Vegetal (W. Larcher, ed.). Editora Rima, São Carlos, p.449-454.

PUHE, J., PERSSON, H. \& BOERJESSON, I. 1986. Root growth and root damages in Scandinavian coniferous forests. Allgemeine Forstzeitschrift 41:488-492.

READ, D.J. 1991. Mycorrhizas in ecosystems. Experientia 47:376-391

SANTOS, O.M. \& VINHA, S.O. 1982. Ocorrência de micorrizas em árvores nativas do sul da Bahia. 1. Estação Ecológica do pau-brasil. Theobroma 12:261-265.

SCHENCK, N.C. \& PEREZ, Y. 1988. Manual for the identification of vesicular arbuscular mycorrhizal fungi. INVAM/University of Florida, Gainesville.

SCHUBERT, A. \& HAYMAN, D.S. 1986. Plant growth responses to MVA: XVI. Effectiveness of different endophytes at different levels of soil phosphate. New Phytologist 103:79-90.

SCHÜEPP, H., DEHN, B. \& STICHER, H. 1987. VA mycorrhiza and heavy metal stress. Angewandte Botanik 61:85-96.

SMA. 1990. The Rain-Forest of the Serra do Mar: degradation and reconstitution. Documents Series, Secretaria do Meio Ambiente, São Paulo.

TRAPPE, J.M. \& SCHENCK, N.C. 1982. Vesicular-arbuscular mycorrhizal fungi (Endogonales). In Methods and principles of mycorrhizal research (N.C. Schenck, ed.). The American Phytopathological Society, St. Paul, p.1-21.

TRUFEM, S.F.B. 1990a. Aspectos ecológicos de fungos micorrízicos vesículo-arbusculares da mata tropical úmida da Ilha do Cardoso, SP, Brasil. Acta Botanica Brasilica 4:31-45.

TRUFEM, S.F.B. 1990b. Aspectos ecológicos de fungos micorrízicos vesículo-arbusculares em ecossistema de duna do parque Estadual da Ilha do Cardoso, São Paulo, Brasil. Aciesp 3:478-497.

TRUFEM, S.F.B. \& MALATINSKY, S.M.M. 1995. Fungos micorrízicos vesículo-arbusculares de Melastomataceae e outras plantas resistentes e sensíveis à poluição na Reserva Biológica do Alto da Serra de Paranapiacaba, SP, Brasil. Hoehnea 22:79-91.

TRUFEM, S.F.B. \& VIRIATO, A. 1990. Fungos micorrízicos vesículo-arbusculares da Reserva Biológica do Alto da Serra de Paranapiacaba, SP, Brasil. Revista Brasileira de Botânica 13:49-54.

TRUFEM, S.F.B., MALATINSKY, S.M.M. \& OTOMO, H.S. 1994. Fungos micorrízicos arbusculares em rizosferas de plantas do litoral arenoso do Parque Estadual da Ilha do Cardoso, SP, Brasil. 2. Acta Botanica Brasilica 8:219-229.

VAVOULIDOU-THEODOROU, E. \& BABEL, V. 1987. On root production in forests: methods and results. Allgemeine Forst-und Jagdzeitung 157:232-238. 
VILARIÑO, A., ARINES, J. \& SCHÜEPP, H. 1992. Propagule production by VA fungi in red clover plants subjected to periodic removal of the aerial parts. In Mycorrhizas in ecosystems (D.J. Read, D.H. Lewis, A.H. Fitter \& I.J. Alexander, eds.). C.B.A. International, Cambridge, p.407.
WANG, G., STRIBLEY, D.P., TINKER, P.B. \& WANG, C. 1985. Soil $\mathrm{pH}$ and vesicular-arbuscular mycorrhizas. In Ecological interactions in soil: plants, microbes and animals (A.H. Fitter, ed.). Blackwell, Oxford, p.219-224. 